\title{
WEIGHTS FOR COMMUTATORS OF THE ONE-SIDED DISCRETE SQUARE FUNCTION, THE WEYL FRACTIONAL INTEGRAL AND OTHER ONE-SIDED OPERATORS.
}

\author{
M. Lorente And M.S. Riveros
}

\begin{abstract}
The purpose of this paper is to prove strong type inequalities with one-sided weights for commutators (with symbol $b \in B M O$ ) of several one-sided operators, such as the one-sided discrete square function, the one-sided fractional operators, or one-sided maximal operators given by the convolution with a smooth function. We also prove that $b \in B M O$ is a necessary condition for the boundedness of commutators of these onesided operators.
\end{abstract}

\section{INTRODUCTION}

There is a great amount of works that deal with the topic of commutators of different operators with BMO functions. If $T$ is the operator given by convolution with a kernel $K$, the commutator of $T$, with a locally integrable function $b$, called the symbol, is the operator

$$
T_{b} f(x)=\int_{\mathbb{R}^{n}}(b(x)-b(y)) K(x-y) f(y) d y .
$$

Sometimes the symbol appears inside the integral with absolute values (see Section 2 for precise definitions).

In 1976, Coifman, Rochberg and Weiss, [4], introduced the higher order commutators,

$$
T_{b}^{k} f(x)=\int_{\mathbb{R}^{n}}(b(x)-b(y))^{k} K(x-y) f(y) d y, \quad k=0,1,2, \ldots
$$

Nottice that $T_{b}^{0}=T$. In [4], it is proved that if $K$ is a Calderón-Zygmund kernel then $T_{b}^{k}$ is bounded from $L^{p}\left(\mathbb{R}^{n}\right)$ to $L^{p}\left(\mathbb{R}^{n}\right)$, for $1<p<\infty$ when $b \in B M O$. In fact, the condition $b \in B M O$ is also necessary in order to have $T_{b}$ bounded on $L^{p}\left(\mathbb{R}^{n}\right)$.

Later, many authors have studied strong and weak type inequalities for commutators with weights (see [3], [13], [14]). Furthermore, many of the

2000 Mathematics Subject Classification. Primary 42B20.

Key words and phrases. One-sided weights, one-sided discrete square function, onesided fractional operators, one-sided maximal functions, commutators.

This research has been supported by Ministerio de Ciencia y Tecnología (BFM 20011638), Junta de Andalucía Universidad Nacional de Córdoba and CONICET. 
results have been generalized to commutators of other operators, not only Calderón-Zygmund operators (see [16], [17], [18]).

In this paper we are interested in studying commutators of certain onesided operators, such as the one-sided discrete square function that appears in [19], the fractional integrals of Weyl and Riemann-Liouville and a class of one-sided maximal operators given by the convolution with a smooth function. This work is highly inspired in the works of C. Segovia and J. L. Torrea [17] and [18]. The main tools for proving the weighted inequalities for these one-sided operators are extrapolation theorems proved by $\mathrm{R}$. Macías and M.S. Riveros in [6]. We also prove that the condition $b \in B M O$ is necessary, i.e., even though we have one-sided operators and our weights are one-sided $A_{p}$ weights, the condition $b \in B M O$ can't be weakened (is a two-sided condition).

Throughout this paper the letter $C$ will be a positive constant, not necessarily the same at each occurrence. If $1 \leq p \leq \infty$, then its conjugate exponent will be denoted by $p^{\prime}$ and $A_{p}$ will be the classical Muckenhoupt's class of weights (see [12]).

\section{Definitions And Statement of the Results}

Definition 2.1. For $f$ locally integrable, we define the one-sided discrete square function applied to $f$ by

$$
S^{+} f(x)=\left(\sum_{n \in \mathbb{Z}}\left|A_{n} f(x)-A_{n-1} f(x)\right|^{2}\right)^{1 / 2}
$$

where $A_{n} f(x)=\frac{1}{2^{n}} \int_{x}^{x+2^{n}} f(y) d y$.

It is not difficult to see that $S^{+} f(x)=\left\|U^{+} f(x)\right\|_{\ell^{2}}$, where $U^{+}$is the sequence valued operator

$$
U^{+} f(x)=\int_{\mathbb{R}} H(x-y) f(y) d y,
$$

where

$$
H(x)=\left\{\frac{1}{2^{n}} \chi_{\left(-2^{n}, 0\right)}(x)-\frac{1}{2^{n-1}} \chi_{\left(-2^{n-1}, 0\right)}(x)\right\}_{n \in \mathbb{Z}} .
$$

(See [19].)

Definition 2.2. The one-sided Hardy-Littlewood maximal operators $M^{+}$ and $M^{-}$are defined for locally integrable functions $f$ by

$$
M^{+} f(x)=\sup _{h>0} \frac{1}{h} \int_{x}^{x+h}|f| \text { and } M^{-} f(x)=\sup _{h>0} \frac{1}{h} \int_{x-h}^{x}|f| .
$$

The good weights for these operators are the one-sided weights, $A_{p}^{+}$and $A_{p}^{-}$:

$$
\sup _{a<b<c} \frac{1}{(c-a)^{p}} \int_{a}^{b} \omega\left(\int_{b}^{c} \omega^{1-p^{\prime}}\right)^{p-1}<\infty, \quad 1<p<\infty
$$


$\left(A_{1}^{+}\right)$

$$
M^{-} \omega(x) \leq C \omega(x) \quad \text { a.e. }
$$

and

$$
A_{\infty}^{+}=\cup_{p \geq 1} A_{p}^{+} .
$$

The classes $A_{p}^{-}$are defined in a similar way. It is interesting to note that $A_{p}=A_{p}^{+} \cap A_{p}^{-}, A_{p} \subsetneq A_{p}^{+}$and $A_{p} \subsetneq A_{p}^{-}$. (See [15], [7], [8], [9] for more definitions and results.)

It is proved in [19], that $\omega \in A_{p}^{+}, 1<p<\infty$, if, and only if, $S^{+}$is bounded from $L^{p}(\omega)$ to $L^{p}(\omega)$ and that $\omega \in A_{1}^{+}$, if, and only if, $S^{+}$is of weak-type $(1,1)$ with respect to $\omega$.

We shall also use for our purposes the following variant of the one-sided Hardy-Littlewood maximal operator:

Definition 2.3. Let $\varphi \in \mathcal{C}_{c}^{\infty}(-\infty, 0], \varphi \geq 0$ nondecreasing in $(-\infty, 0]$. For $\varepsilon>0$ let $\varphi_{\varepsilon}(x)=\varepsilon^{-1} \varphi\left(\varepsilon^{-1} x\right)$. The maximal operator associated to $\varphi$ is defined by

$$
M_{\varphi}^{+} f(x)=\sup _{\varepsilon>0} \varphi_{\varepsilon} *|f|(x)
$$

It is not difficult to see that $M_{\varphi}^{+} f$ is pointwise equivalent to $M^{+} f$. As a consequence, $M_{\varphi}^{+}$is bounded from $L^{p}(\omega)$ to $L^{p}(\omega)$, for $1<p<\infty$ and $\omega \in A_{p}^{+}$.

Definition 2.4. The one-sided maximal fractional operator $M_{\alpha}^{+}, 0<\alpha<$ 1 , is defined, for locally integrable functions $f$, by

$$
M_{\alpha}^{+} f(x)=\sup _{h>0} \frac{1}{h^{1-\alpha}} \int_{x}^{x+h}|f| .
$$

It is proved in [2] that $\left\|\left(M_{\alpha}^{+} f\right) \omega\right\|_{q} \leq C\|f \omega\|_{p}$ if and only if $\omega \in$ $A^{+}(p, q)$, for $1<p<q, 1 / p-1 / q=\alpha$, where

$$
\left(\frac{1}{h} \int_{x-h}^{x} \omega^{q}\right)^{1 / q}\left(\frac{1}{h} \int_{x}^{x+h} \omega^{-p^{\prime}}\right)^{1 / p^{\prime}} \leq C,
$$

$$
\left\|\omega \chi_{[x-h, x]}\right\|_{\infty}\left(\frac{1}{h} \int_{x}^{x+h} \omega^{-p^{\prime}}\right)^{1 / p^{\prime}} \leq C
$$

for all $h>0$ and $x \in \mathbb{R}$.

We also have a variant of the operator $M_{\alpha}^{+}$:

Definition 2.5. Let $0<\alpha<1$ and let $\varphi_{\alpha} \in \mathcal{C}^{\infty}((-\infty, 0]), \varphi_{\alpha} \geq 0$, nondecreasing in $(-\infty, 0]$ and such that $\left|\varphi_{\alpha}(x-y)-\varphi_{\alpha}(x)\right| \leq C|y||x|^{-2+\alpha}$, for all $x, y$ such that $|x|>2|y|$. For each $\varepsilon>0$, set $\varphi_{\alpha, \varepsilon}(x)=\varepsilon^{-1+\alpha} \varphi_{\alpha}\left(\varepsilon^{-1} x\right)$. We define the maximal operator associated to $\varphi_{\alpha}$ by

$$
M_{\varphi_{\alpha}}^{+} f(x)=\sup _{\varepsilon>0} \varphi_{\alpha, \varepsilon} *|f|(x) .
$$

It is very easy to see that $M_{\varphi_{\alpha}}^{+} f(x) \leq C M_{\alpha}^{+} f(x)$. 
Definition 2.6. Let $b \in L_{l o c}^{1}(\mathbb{R})$. We say that $b \in B M O$ if

$$
\|b\|_{B M O}=\sup _{I} \frac{1}{|I|} \int_{I}\left|b-b_{I}\right|<\infty,
$$

where $I$ denotes any bounded interval and $b_{I}=\frac{1}{|I|} \int_{I} b$.

Definition 2.7. Let $f$ be a locally integrable function. The one-sided sharp maximal function is defined by

$$
f^{\#,+}(x)=\sup _{h>0} \frac{1}{h} \int_{x}^{x+h}\left(f(y)-\frac{1}{h} \int_{x+h}^{x+2 h} f\right)^{+} d y .
$$

It is proved in [11] that

$$
\begin{aligned}
f^{\#,+}(x) & \leq \sup _{h>0} \inf _{a \in \mathbb{R}} \frac{1}{h} \int_{x}^{x+h}(f(y)-a)^{+} d y+\frac{1}{h} \int_{x+h}^{x+2 h}(a-f(y))^{+} d y \\
& \leq C\|f\|_{B M O} .
\end{aligned}
$$

Now we shall state our results.

Theorem 2.8. Let $b \in B M O, H$ as in (2.2) and $k=0,1, \ldots$ The $k$-th order commutator of the one-sided discrete square function is defined by

$$
S_{b}^{+, k} f(x)=\left\|\int_{\mathbb{R}}(b(x)-b(y))^{k} H(x-y) f(y) d y\right\|_{\ell^{2}} .
$$

Then for $1<p<\infty$ and $\omega \in A_{p}^{+}$,

$$
\int_{\mathbb{R}}\left|S_{b}^{+, k} f\right|^{p} \omega \leq C \int_{\mathbb{R}}|f|^{p} \omega,
$$

for all bounded functions $f$ with compact support.

Theorem 2.9. Let $0<\alpha<1, b \in B M O$, and $k=0,1, \ldots$ The $k$-th order commutator of the Weyl fractional integral is defined by

$$
I_{\alpha, b}^{+, k} f(x)=\int_{x}^{\infty}(b(x)-b(y))^{k} \frac{f(y)}{(y-x)^{1-\alpha}} d y .
$$

(The Weyl fractional integral is the corresponding one for $k=0$ ) Then for all $\omega \in A^{+}(p, q), 1<p<q<\infty, 1 / p-1 / q=\alpha$, we have

$$
\left(\int_{\mathbb{R}}\left|I_{\alpha, b}^{+, k} f\right|^{q} \omega^{q}\right)^{1 / q} \leq C\left(\int_{\mathbb{R}}|f|^{p} \omega^{p}\right)^{1 / p}
$$

for all bounded $f$ with compact support.

In the following theorems we prove that for commutators of one-sided operators given by convolution with a smooth function, $b \in B M O$ is also a necessary condition in order to have the commutator bounded on $L^{p}(\omega)$.

Let $\varphi \in \mathcal{C}_{c}^{\infty}(-\infty, 0], \varphi \geq 0$ nondecreasing in $(-\infty, 0]$. Then it is easy to see that there exists $C>0$ such that $|\varphi(x-y)-\varphi(x)| \leq C|y||x|^{-2}$, for all $x, y$ such that $|x|>2|y|$.

For $k=0,1, \ldots$, the $k$-th order commutator of $M_{\varphi}^{+}$with symbol $b$ is defined by

$$
M_{\varphi, b}^{+, k} f(x)=\sup _{\varepsilon>0} \int_{x}^{\infty}|b(x)-b(y)|^{k} \varphi_{\varepsilon}(x-y)|f(y)| d y .
$$


Theorem 2.10. Let $1<p<\infty, b \in B M O$ and $\omega \in A_{p}^{+}$. Then

$$
\int_{\mathbb{R}}\left|M_{\varphi, b}^{+, k} f\right|^{p} \omega \leq C \int_{\mathbb{R}}|f|^{p} \omega .
$$

If we consider $\varphi$ as before and such that $\chi_{[-1,0]} \leq \varphi$, then we have

$$
M_{b}^{+, k} f(x) \leq M_{\varphi, b}^{+, k} f(x)
$$

where

$$
M_{b}^{+, k} f(x)=\sup _{h>0} \frac{1}{h} \int_{x}^{x+h}|b(x)-b(y)|^{k}|f(y)| d y .
$$

Therefore, if $b \in B M O$, Theorem 2.10 gives that $M_{b}^{+, k}$ is bounded from $L^{p}(\omega)$ to $L^{p}(\omega)$, for $1<p<\infty$ and $\omega \in A_{p}^{+}$. In fact, more can be said, the converse is also true.

Theorem 2.11. The following conditions are equivalent:

(i) $M_{b}^{+, k}$ is bounded from $L^{p}(\omega)$ to $L^{p}(\omega)$, for all $p$ with $1<p<\infty$ and $\omega \in A_{p}^{+}$.

(ii) $M_{b}^{+, k}$ is bounded from $L^{p}(d x)$ to $L^{p}(d x)$, for some $p>1$.

(iii) $b \in B M O$.

Analogous results hold for $M_{\varphi_{\alpha}}^{+}$. Let $0<\alpha<1$. Suppose $\varphi_{\alpha} \in$ $\mathcal{C}^{\infty}((-\infty, 0]), \varphi_{\alpha} \geq 0$, nondecreasing in $(-\infty, 0]$ and such that $\left|\varphi_{\alpha}(x-y)-\varphi_{\alpha}(x)\right| \leq C|y||x|^{\alpha-2}$, for all $x, y$ such that $|x|>2|y|$.

For $k=0,1, \ldots$, the $k$-th order commutator of $M_{\varphi_{\alpha}}^{+}$with symbol $b$ is defined by

$$
M_{\varphi_{\alpha}, b}^{+, k} f(x)=\sup _{\varepsilon>0} \int_{x}^{\infty}|b(x)-b(y)|^{k} \varphi_{\alpha, \varepsilon}(x-y)|f(y)| d y .
$$

Then we have the following:

Theorem 2.12. Let $b \in B M O$ and let $(p, q)$ be such that $1<p<q<\infty$ and $\frac{1}{p}-\frac{1}{q}=\alpha$. Then, for all $\omega \in A^{+}(p, q)$, we have that

$$
\left(\int_{\mathbb{R}}\left|M_{\varphi_{\alpha}, b}^{+, k} f\right|^{q} \omega^{q}\right)^{1 / q} \leq C\left(\int_{\mathbb{R}}|f|^{p} \omega^{p}\right)^{1 / p} .
$$

Since $M_{\varphi_{\alpha}}^{+} f(x) \leq C M_{\alpha}^{+} f(x)$, the case $k=0$ is a consequence of a result of Andersen and Sawyer [2]. And if we choose $\varphi_{\alpha}$ such that $\chi_{[-1,0]} \leq \varphi_{\alpha}$, then $M_{\alpha, b}^{+, k} f(x) \leq M_{\varphi_{\alpha, b}}^{+, k} f(x)$, where

$$
M_{\alpha, b}^{+, k} f(x)=\sup _{h>0} \frac{1}{h^{1-\alpha}} \int_{x}^{x+h}|b(x)-b(y)|^{k}|f(y)| d y .
$$

For this operator we can also prove that $b \in B M O$ is a necessary condition.

Theorem 2.13. The following conditions are equivalent:

(i) $M_{\alpha, b}^{+, k}$ is bounded from $L^{p}\left(\omega^{p}\right)$ to $L^{q}\left(\omega^{q}\right)$ for all pairs $(p, q)$ such that $\frac{1}{p}-\frac{1}{q}=\alpha, 1<p<q<\infty$ and $\omega \in A^{+}(p, q)$.

(ii) $M_{\alpha, b}^{+, k}$ is bounded from $L^{p}(d x)$ to $L^{q}(d x)$ for some pair $(p, q)$ such that $\frac{1}{p}-\frac{1}{q}=\alpha$ and $1<p<q<\infty$.

(iii) $b \in B M O$. 


\section{Proof of the Results}

The main tools for proving our results are two extrapolation theorems that appeared in [6], with slight modifications.

Theorem 3.1. Let $T$ be a sublinear operator defined in $\mathcal{C}_{c}^{\infty}(\mathbb{R})$. Assume that for all $\omega$ such that $\omega^{-1} \in A_{1}^{-}$, there exists $C=C(\omega)$ such that

$$
\|\omega T f\|_{\infty} \leq C\|f \omega\|_{\infty}
$$

Then, for all $\omega \in A_{p}^{+}, 1<p<\infty$, there exists $C=C(\omega)$ such that

$$
\left(\int|T f|^{p} \omega\right)^{1 / p} \leq C\left(\int|f|^{p} \omega\right)^{1 / p}
$$

provided that the left hand side is finite.

Theorem 3.2. Let $1<p_{0}<\infty$ and $T$ be a sublinear operator defined in $\mathcal{C}_{c}^{\infty}(\mathbb{R})$. Assume that for all $\omega \in A^{+}\left(p_{0}, \infty\right)$ there exists $C=C(\omega)$ such that

$$
\|\omega T f\|_{\infty} \leq C\|f \omega\|_{p_{0}} .
$$

Then, for all pairs $(p, q)$ such that $1<p<p_{0}, \frac{1}{p}-\frac{1}{q}=\frac{1}{p_{0}}$ and all $\omega \in A^{+}(p, q)$, there exists $C=C(\omega)$ such that

$$
\|\omega T f\|_{q} \leq C\|f \omega\|_{p}
$$

provided that the left hand side is finite.

We will also need the following result of Martín-Reyes and de la Torre (theorem 4 in [11]):

Theorem 3.3. Let $1<p<\infty$. If $\omega \in A_{p}^{+}$and $M^{+} f \in L^{p}(\omega)$, then there exists $C=C(w)$ such that

$$
\int_{\mathbb{R}}\left(M^{+} f\right)^{p} \omega \leq C \int_{\mathbb{R}}\left(f^{\#,+}\right)^{p} \omega .
$$

An other result that will be used often is the following (see [15]):

Theorem 3.4. Let $\omega \in A_{1}^{-}$. Then there exists $s>1$ such that $\omega^{r} \in A_{1}^{-}$, for all $r$ such that $1<r \leq s$.

Proof of Theorem 2.8. Let $\omega \in A_{p}^{+}$. For $b \in L^{\infty} \subset B M O$ and $f$ bounded of compact support, we have that $S_{b}^{+, k} f \in L^{p}(\omega)$. Then, by Theorem 3.3,

$$
\int_{\mathbb{R}}\left|S_{b}^{+, k} f\right|^{p} \omega \leq C \int_{\mathbb{R}}\left|M^{+}\left(S_{b}^{+, k} f\right)\right|^{p} \omega \leq C \int_{\mathbb{R}}\left|\left(S_{b}^{+, k} f\right)^{\#,+}\right|^{p} \omega .
$$

To prove the theorem for any $b \in B M O$ we proceed in the same way as in [5]. We will control $\left(S_{b}^{+, k} f\right)^{\#,+}$ by some one-sided maximal operators. Using Theorem 3.1, we shall prove that they are bounded from $L^{p}(\omega)$ to $L^{p}(\omega)$. 
Let $\lambda$ be an arbitrary constant. Then $b(x)-b(y)=(b(x)-\lambda)-(b(y)-\lambda)$ and

$$
\begin{gathered}
S_{b}^{+, k} f(x)=\left\|\int_{\mathbb{R}}(b(x)-b(y))^{k} H(x-y) f(y) d y\right\|_{\ell^{2}} \\
=\left\|\sum_{j=0}^{k} C_{j, k}(b(x)-\lambda)^{j} \int_{\mathbb{R}}(b(y)-\lambda)^{k-j} H(x-y) f(y) d y\right\|_{\ell^{2}} \\
\leq\left\|\int_{\mathbb{R}}(b(y)-\lambda)^{k} H(x-y) f(y) d y\right\|_{\ell^{2}} \\
+\left\|\sum_{j=1}^{k} C_{j, k}(b(x)-\lambda)^{j} \int_{\mathbb{R}}(b(y)-\lambda)^{k-j} H(x-y) f(y) d y\right\|_{\ell^{2}} \\
=S^{+}\left((b-\lambda)^{k} f\right)(x) \\
+\left\|\sum_{j=1}^{k} \sum_{s=0}^{k-j} C_{j, k, s}(b(x)-\lambda)^{s+j} \int_{\mathbb{R}}(b(x)-b(y))^{k-j-s} H(x-y) f(y) d y\right\|_{\ell^{2}} \\
\leq S^{+}\left((b-\lambda)^{k} f\right)(x)+\sum_{m=0}^{k-1} C_{k, m}|b(x)-\lambda|^{k-m} S_{b}^{+, m} f(x),
\end{gathered}
$$

where $C_{j, k}$ (respectively $C_{j, k, s}$ ) are absolute constants depending only on $j$ and $k$ (respectively $j, k$ and $s$ ). Let $x \in \mathbb{R}, h>0$. Let $i \in \mathbb{Z}$ be such that $2^{i} \leq h<2^{i+1}$ and set $J=\left[x, x+2^{i+3}\right]$. Then, write $f=f_{1}+f_{2}$, where $f_{1}=f \chi_{J}$ and set $\lambda=b_{J}$. Then

$$
\begin{aligned}
& \frac{1}{h} \int_{x}^{x+2 h}\left|S_{b}^{+, k} f(y)-S^{+}\left(\left(b-b_{J}\right)^{k} f_{2}\right)(x)\right| d y \\
& \leq \frac{1}{h} \int_{x}^{x+2 h}\left|S^{+}\left(\left(b-b_{J}\right)^{k} f_{1}\right)(y)\right| d y \\
& \quad+\frac{1}{h} \int_{x}^{x+2 h}\left|S^{+}\left(\left(b-b_{J}\right)^{k} f_{2}\right)(y)-S^{+}\left(\left(b-b_{J}\right)^{k} f_{2}\right)(x)\right| d y \\
& \quad+\sum_{m=0}^{k-1} C_{k, m} \frac{1}{h} \int_{x}^{x+2 h}\left|b(y)-b_{J}\right|^{k-m}\left|S_{b}^{+, m} f(y)\right| d y \\
& =I(x)+I I(x)+\operatorname{III}(x) .
\end{aligned}
$$

Let $U^{+}$be as in $(2.1)$. Then

$$
I I(x) \leq \frac{1}{h} \int_{x}^{x+2^{i+3}}\left\|U^{+}\left(\left(b-b_{J}\right)^{k} f_{2}\right)(y)-U^{+}\left(\left(b-b_{J}\right)^{k} f_{2}\right)(x)\right\|_{\ell^{2}} d y
$$

and

$$
\begin{aligned}
& \left\|U^{+}\left(\left(b-b_{J}\right)^{k} f_{2}\right)(y)-U^{+}\left(\left(b-b_{J}\right)^{k} f_{2}\right)(x)\right\|_{\ell^{2}} \\
& \leq \int_{x+2^{i+3}}^{\infty}\left|b(t)-b_{J}\right|^{k} \mid f(t)\|\| H(y-t)-H(x-t) \|_{\ell^{2}} d t .
\end{aligned}
$$


Consider the following sublinear operators defined in $\mathcal{C}_{c}^{\infty}$ :

$$
\begin{gathered}
M_{1}^{+} f(x)=\sup _{i \in \mathbb{Z}} \frac{1}{2^{i}} \int_{x}^{x+2^{i+2}}\left|S^{+}\left(\left(b-b_{J}\right)^{k} f \chi_{J}\right)(y)\right| d y \\
M_{2}^{+} f(x)=\sup _{i \in \mathbb{Z}} \frac{1}{2^{i}} \int_{x}^{x+2^{i+3}} \int_{x+2^{i+3}}^{\infty}\left|b(t)-b_{J}\right|^{k}|f(t)||| H(y-t)-H(x-t) \|_{\ell^{2}} d t d y
\end{gathered}
$$

and

$$
M_{3, m}^{+} f(x)=\sup _{h>0} \frac{1}{h} \int_{x}^{x+2 h}\left|b(y)-b_{[x, x+8 h]}\right|^{k-m}|f(y)| d y, \quad 0 \leq m \leq k-1,
$$

where, for each $i, J$ denotes the interval $\left[x, x+2^{i+3}\right]$.

Inequalities (3.3), (3.4), (3.5) and the above definitions give that

$$
\left(S_{b}^{+, k} f\right)^{\#,+}(x) \leq C\left(M_{1}^{+} f(x)+M_{2}^{+} f(x)+\sum_{m=0}^{k-1} M_{3, m}^{+}\left(S_{b}^{+, m} f\right)(x)\right) .
$$

We shall prove, using Theorem 3.1, that these operators are bounded from $L^{p}(\omega)$ to $L^{p}(\omega), \omega \in A_{p}^{+}, 1<p<\infty$.

Boundedness of $M_{1}^{+}$: Let $\omega$ be a weight such that $\omega^{-1} \in A_{1}^{-}$. Let $1<q<\infty$ and $1<s<\infty$ be such that $\omega^{-q s} \in A_{1}^{-}$(Theorem 3.4). By Hölder's and John-Nirenberg's inequalities and the fact that $S^{+}$is bounded from $L^{q}(d x)$ to $L^{q}(d x)$ we get

$$
\begin{aligned}
& \frac{1}{2^{i}} \int_{x}^{x+2^{i+2}}\left|S^{+}\left(\left(b-b_{J}\right)^{k} f \chi_{J}\right)(y)\right| d y \\
& \leq C\left(\frac{1}{2^{i}} \int_{x}^{x+2^{i+2}}\left|S^{+}\left(\left(b-b_{J}\right)^{k} f \chi_{J}\right)(y)\right|^{q} d y\right)^{1 / q} \\
& \leq C\left(\frac{1}{2^{i}} \int_{x}^{x+2^{i+3}}\left|\left(b(y)-b_{J}\right)^{k} f(y)\right|^{q} d y\right)^{1 / q} \\
& \leq C\|f \omega\|_{\infty}\left(\frac{1}{2^{i}} \int_{x}^{x+2^{i+3}}\left|\left(b(y)-b_{J}\right)^{k} \omega^{-1}(y)\right|^{q} d y\right)^{1 / q} \\
& \leq C\|f \omega\|_{\infty}\left(\frac{1}{2^{i}} \int_{x}^{x+2^{i+3}}\left|b-b_{J}\right|^{k q s^{\prime}}\right)^{1 / q s^{\prime}}\left(\frac{1}{2^{i}} \int_{x}^{x+2^{i+3}} \omega^{-q s}\right)^{1 / s q} \\
& \leq C\|f \omega\|_{\infty}\|b\|_{B M O}^{k} \omega^{-1}(x) \text {. }
\end{aligned}
$$

Then, for all $\omega$ such that $\omega^{-1} \in A_{1}^{-}$,

$$
\left\|\omega M_{1}^{+} f\right\|_{\infty} \leq C\|b\|_{B M O}^{k}\|f \omega\|_{\infty},
$$

and by Theorem 3.1, we obtain that for all $\omega \in A_{p}^{+}, 1<p<\infty$,

$$
\left\|M_{1}^{+} f\right\|_{\omega, p} \leq C\|b\|_{B M O}^{k}\|f\|_{\omega, p} .
$$


Boundedness of $M_{2}^{+}$: Let $\omega$ be such that $\omega^{-1} \in A_{1}^{-}$. Then, if $I_{j}=$ $\left[x, x+2^{j+1}\right]$, we have that

$$
\begin{aligned}
& \int_{x+2^{i+3}}^{\infty}\left|b(t)-b_{J}\right|^{k}|f(t)||| H(y-t)-H(x-t) \|_{\ell^{2}} d t \\
& \leq C \sum_{j=i+3}^{\infty} \int_{x+2^{j}}^{x+2^{j+1}}\left|b(t)-b_{I_{j}}\right|^{k}|f(t)|\|H(y-t)-H(x-t)\|_{\ell^{2}} d t \\
& \quad+C \sum_{j=i+3}^{\infty}\left|b_{I_{j}}-b_{J}\right|^{k} \int_{x+2^{j}}^{x+2^{j+1}}|f(t)||| H(y-t)-H(x-t) \|_{\ell^{2}} d t \\
& =I V(x)+V(x) .
\end{aligned}
$$

We choose $\left(s, s^{\prime}\right)$ and $\left(t, t^{\prime}\right)$ such that $\omega^{-s} \in A_{1}^{-}$and $\omega^{-s t^{\prime}} \in A_{1}^{-}$. Then, by Hölder's inequality with exponents $\left(s, s^{\prime}\right)$ and $\left(t, t^{\prime}\right)$,

$$
\begin{aligned}
I V(x) \leq C \sum_{j=i+3}^{\infty} & \left(\int_{I_{j}}\left|b-b_{I_{j}}\right|^{k s} \omega^{-s} \omega^{s}|f|^{s}\right)^{1 / s} \\
& \times\left(\int_{x+2^{j}}^{x+2^{j+1}}\|H(y-t)-H(x-t)\|_{\ell^{2}}^{s^{\prime}} d t\right)^{1 / s^{\prime}} \\
\leq C\|f \omega\|_{\infty} & \sum_{j=i+3}^{\infty}\left(\int_{I_{j}}\left|b-b_{I_{j}}\right|^{k s t}\right)^{1 / s t}\left(\int_{I_{j}} \omega^{-s t^{\prime}}\right)^{1 / s t^{\prime}} \\
\times & \left(\int_{x+2^{j}}^{x+2^{j+1}}\|H(y-t)-H(x-t)\|_{\ell^{2}}^{s^{\prime}} d t\right)^{1 / s^{\prime}}
\end{aligned}
$$

It is proved in theorem 1.6 of [19] that for all $y \in\left[x, x+2^{i+3}\right]$ the kernel $H$ satisfies

$$
\left(\int_{x+2^{j}}^{x+2^{j+1}}\|H(y-t)-H(x-t)\|_{\ell^{2}}^{s^{\prime}} d t\right)^{1 / s^{\prime}} \leq C \frac{2^{i / s^{\prime}}}{2^{j}}
$$

Then, using that $b \in B M O$, the fact that $\omega^{-s t^{\prime}} \in A_{1}^{-}$and (3.9), we get

$$
\begin{aligned}
I V(x) & \leq C\|f \omega\|_{\infty} \sum_{j=i+3}^{\infty}\|b\|_{B M O}^{k}\left(2^{j}\right)^{1 / s t} \omega^{-1}(x)\left(2^{j+1}\right)^{1 / s t^{\prime}} \frac{2^{i / s^{\prime}}}{2^{j}} \\
& \leq C \omega^{-1}(x)\|f \omega\|_{\infty}\|b\|_{B M O}^{k} \sum_{j=i+3}^{\infty}\left(\frac{2^{i}}{2^{j}}\right)^{1 / s^{\prime}} \\
& \leq C \omega^{-1}(x)\|f \omega\|_{\infty}\|b\|_{B M O}^{k} .
\end{aligned}
$$

Using again Hölder's inequality, (3.9), the fact that $w^{-s} \in A_{1}^{-}$and lemma 
1 in [5], we get

$$
\begin{aligned}
& V(x)= C \sum_{j=i+3}^{\infty}\left|b_{I_{j}}-b_{J}\right|^{k} \int_{x+2^{j}}^{x+2^{j+1}}|f(t)|\|H(y-t)-H(x-t)\|_{\ell^{2}} d t \\
& \leq C \sum_{j=i+3}^{\infty}\|b\|_{B M O}^{k}(2(j-i))^{k}\|f \omega\|_{\infty}\left(\int_{I_{j}} \omega^{-s}\right)^{1 / s} \\
& \times\left(\int_{x+2^{j}}^{x+2^{j+1}}\|H(y-t)-H(x-t)\|_{\ell^{\prime}}^{s^{\prime}} d t\right)^{1 / s^{\prime}} \\
& \leq C\|b\|_{B M O}^{k}\|f \omega\|_{\infty} \omega^{-1}(x) \sum_{j=i+3}^{\infty}(2(j-i))^{k}\left(2^{j+1}\right)^{1 / s} \frac{2^{i / s^{\prime}}}{2^{j}} \\
& \leq C \omega^{-1}(x)\|b\|_{B M O}^{k}\|f \omega\|_{\infty} \sum_{j=i+3}^{\infty} \frac{(j-i)^{k} 2^{i / s^{\prime}}}{2^{j / s^{\prime}}} \\
& \leq C \omega^{-1}(x)\|b\|_{B M O}^{k}\|f \omega\|_{\infty} .
\end{aligned}
$$

Then, by (3.7), (3.10) and (3.11), we get that, for all $\omega$ such that $\omega^{-1} \in A_{1}^{-}$,

$$
\left\|\omega M_{2}^{+} f\right\|_{\infty} \leq C\|f \omega\|_{\infty} .
$$

Then, by Theorem 3.1, for all $1<p<\infty$ and $\omega \in A_{p}^{+}$,

$$
\left\|M_{2}^{+} f\right\|_{p, \omega} \leq C\|f\|_{p, \omega} .
$$

Boundedness of $M_{3, m}^{+}$: Let $\omega$ be such that $\omega^{-1} \in A_{1}^{-}$and let $q>1$ be such that $\omega^{-q} \in A_{1}^{-}$. Then, using again Hölder's and John-Nirenberg's inequalities we obtain

$$
\begin{aligned}
& \frac{1}{h} \int_{x}^{x+2 h}\left|b(y)-b_{[x, x+8 h]}\right|^{k-m}|f(y)| d y \\
\leq & \left(\frac{1}{h} \int_{x}^{x+2 h}\left|b(y)-b_{[x, x+8 h]}\right|^{(k-m) q^{\prime}} d y\right)^{1 / q^{\prime}}\left(\frac{1}{h} \int_{x}^{x+2 h}|f(y)|^{q} d y\right)^{1 / q} \\
\leq & C\|b\|_{B M O}^{k-m}\left(\frac{1}{h} \int_{x}^{x+2 h}|f(y)|^{q} d y\right)^{1 / q} \\
\leq & C\|b\|_{B M O}^{k-m}\|f \omega\|_{\infty}\left(\frac{1}{h} \int_{x}^{x+2 h} \omega^{-q} d y\right)^{1 / q} \\
\leq & C\|b\|_{B M O}^{k-m}\|f \omega\|_{\infty} \omega^{-1}(x) .
\end{aligned}
$$

Then, for all $\omega$ such that $\omega^{-1} \in A_{1}^{-}$,

$$
\left\|\omega M_{3, m}^{+} f\right\|_{\infty} \leq C\|b\|_{B M O}^{k-m}\|\omega f\|_{\infty} .
$$


Therefore, by Theorem 3.1, we have that, for all $\omega \in A_{p}^{+}, 1<p<\infty$,

$$
\left\|M_{3, m}^{+} f\right\|_{\omega, p} \leq C\|b\|_{B M O}^{k-m}\|f\|_{\omega, p} .
$$

Using now the induction principle (the case $k=0$ was proved in [19]), we obtain that, for all $\omega \in A_{p}^{+}, 1<p<\infty$,

$$
\left\|M_{3, m}^{+}\left(S_{b}^{+, m} f\right)\right\|_{\omega, p} \leq C\|b\|_{B M O}^{k-m}\left\|S_{b}^{+, m} f\right\|_{\omega, p} \leq C\|b\|_{B M O}^{k}\|f\|_{\omega, p} .
$$

Proof of Theorem 2.9. This proof follows the same pattern as the preceding one. Let $b \in B M O$ bounded, and $\lambda \in \mathbb{R}$, then, as in (3.2), we can write

$$
I_{\alpha, b}^{k,+} f(x)=I_{\alpha}^{+}\left((b-\lambda)^{k} f\right)(x)+\sum_{m=0}^{k-1} C_{k, m}(b(x)-\lambda)^{k-m} I_{\alpha, b}^{+, m} f(x) .
$$

Let $x \in \mathbb{R}, h>0$ and $J=[x, x+4 h]$. Write $f=f_{1}+f_{2}$, where $f_{1}=f \chi_{J}$ and set $\lambda=b_{J}$. Then,

$$
\begin{aligned}
& \frac{1}{h} \int_{x}^{x+2 h}\left|I_{\alpha, b}^{+, k} f(y)-I_{\alpha}^{+}\left(\left(b-b_{J}\right)^{k} f_{2}\right)(x+2 h)\right| d y \\
& \leq \frac{1}{h} \int_{x}^{x+2 h}\left|I_{\alpha}^{+}\left(\left(b-b_{J}\right)^{k} f_{1}\right)(y)\right| d y \\
& \quad+\frac{1}{h} \int_{x}^{x+2 h}\left|I_{\alpha}^{+}\left(\left(b-b_{J}\right)^{k} f_{2}\right)(y)-I_{\alpha}^{+}\left(\left(b-b_{J}\right)^{k} f_{2}\right)(x+2 h)\right| d y \\
& \quad+\sum_{m=0}^{k-1} C_{k, m} \frac{1}{h} \int_{x}^{x+2 h}\left|b(y)-b_{J}\right|^{k-m}\left|I_{\alpha, b}^{+, m} f(y)\right| d y \\
& =I(x)+I I(x)+I I I(x) .
\end{aligned}
$$

It is clear that

$$
I I I(x) \leq \sum_{m=0}^{k-1} C_{k, m} M_{3, m}^{+}\left(I_{\alpha, b}^{+, m} f\right)(x)
$$

where $M_{3, m}^{+}$is as in the proof of Theorem 2.8. Then, we already know that $M_{3, m}^{+}$is bounded from $L^{p}(\omega)$ to $L^{p}(\omega)$, provided $\omega \in A_{p}^{+}$and $1<p<\infty$. So, if $\omega \in A^{+}(p, q), 1 / p-1 / q=\alpha$, then $\omega^{q} \in A_{q}^{+}$, and by induction (see $\mathrm{k}=0$ in $[10])$, we obtain

$$
\left\|M_{3, m}^{+}\left(I_{\alpha, b}^{+, m} f\right)\right\|_{\omega^{q}, q} \leq C\|b\|_{B M O}^{k-m}\left\|I_{\alpha, b}^{+, m} f\right\|_{\omega^{q}, q} \leq C\|b\|_{B M O}^{k}\|f\|_{\omega^{p}, p},
$$

for all $f \in \mathcal{C}_{c}^{\infty}(\mathbb{R})$.

To control $I(x)$ let us define

$$
M_{4}^{+} f(x)=\sup _{h>0} \frac{1}{h} \int_{x}^{x+2 h}\left|I_{\alpha}^{+}\left(\left(b-b_{J}\right)^{k} f \chi_{J}\right)(y)\right| d y,
$$


where, for each $h>0, J=[x, x+4 h]$. It is not difficult to see that $M_{4}^{+}$ is a sublinear operator in $\mathcal{C}_{c}^{\infty}$ and it is clear that $I(x) \leq M_{4}^{+} f(x)$. Let us prove that $M_{4}^{+}$is bounded from $L^{p}\left(\omega^{p}\right)$ to $L^{q}\left(\omega^{q}\right)$ using Theorem 3.2.

Let $\omega \in A^{+}\left(\frac{1}{\alpha}, \infty\right)$, then $\omega^{\frac{-1}{1-\alpha}} \in A_{1}^{-}$. Therefore, there exist $t_{1}>1$ and $t_{2}>1$ such that $\omega^{\frac{-t_{1}}{1-\alpha}} \in A_{1}^{-}$and $\omega^{\frac{-t_{1} t_{2}}{1-\alpha}} \in A_{1}^{-}$. Let $s>1$ and $r>1$ be such that $s=t_{1} /(1-\alpha)$ and $1 / r-1 / s=\alpha$. Then, using Hölder's inequality and the fact that $I_{\alpha}^{+}$is bounded from $L^{r}(\mathbb{R})$ to $L^{s}(\mathbb{R})$, we get

$$
\begin{aligned}
& \frac{1}{h} \int_{x}^{x+2 h}\left|I_{\alpha}^{+}\left(\left(b-b_{J}\right)^{k} f \chi_{J}\right)(y)\right| d y \\
\leq & \left(\frac{1}{h} \int_{x}^{x+2 h}\left|I_{\alpha}^{+}\left(\left(b-b_{J}\right)^{k} f \chi_{J}\right)(y)\right|^{s} d y\right)^{1 / s} \\
\leq & C h^{\alpha}\left(\frac{1}{h} \int_{x}^{x+4 h}\left|\left(b(y)-b_{J}\right)^{k} f(y)\right|^{r} \omega^{r} \omega^{-r} d y\right)^{1 / r} \\
\leq & C h^{\alpha}\left(\frac{1}{h} \int_{x}^{x+4 h}\left|\left(b-b_{J}\right)^{k}\right|^{r \frac{s}{r}} \omega^{-r \frac{s}{r}}\right)^{\frac{1}{s}}\left(\frac{1}{h} \int_{x}^{x+4 h}|f|^{\frac{1}{\alpha}} \omega^{\frac{1}{\alpha}}\right)^{\alpha} .
\end{aligned}
$$

Therefore, using Hölder's and John-Nirenberg's inequalities and the fact that $\omega^{-s t_{2}} \in A_{1}^{-}$, the chain of inequalities in (3.13) can be continued as follows:

$$
\begin{aligned}
& \leq C\|f \omega\|_{\frac{1}{\alpha}}\left(\frac{1}{h} \int_{x}^{x+4 h}\left|b-b_{J}\right|^{k s} \omega^{-s}\right)^{\frac{1}{s}} \\
& \leq C\|f \omega\|_{\frac{1}{\alpha}}\left(\frac{1}{h} \int_{x}^{x+4 h}\left|b-b_{J}\right|^{k s t_{2}^{\prime}}\right)^{\frac{1}{s t_{2}^{\prime}}}\left(\frac{1}{h} \int_{x}^{x+4 h} \omega^{-s t_{2}}\right)^{\frac{1}{s t_{2}}} \\
& \leq C\|f \omega\|_{\frac{1}{\alpha}}\|b\|_{B M O}^{k} \omega^{-1}(x) .
\end{aligned}
$$

As a consequence,

$$
\left\|\omega M_{4}^{+} f\right\|_{\infty} \leq C\|b\|_{B M O}^{k}\|f \omega\|_{\frac{1}{\alpha}} .
$$

Then, by Theorem 3.2, for all $\omega \in A^{+}(p, q), \frac{1}{p}-\frac{1}{q}=\alpha$,

$$
\left\|M_{4}^{+} f\right\|_{\omega^{q}, q} \leq C\|b\|_{B M O}^{k}\|f\|_{\omega^{p}, p} .
$$

Finally, we shall estimate $I I(x)$. We have that

$$
I I(x)=\frac{1}{h} \int_{x}^{x+2 h}\left|\int_{x+4 h}^{\infty} \sigma(t, y) d t\right| d y
$$

where

$$
\sigma(t, y)=\left(b(t)-b_{J}\right)^{k} f(t)\left(\frac{1}{(y-t)^{1-\alpha}}-\frac{1}{(x+2 h-t)^{1-\alpha}}\right) .
$$


Consider the following sublinear operator in $C_{c}^{\infty}(\mathbb{R})$ :

$$
M_{5}^{+} f(x)=\sup _{h>0} \frac{1}{h} \int_{x}^{x+2 h}\left|\int_{x+4 h}^{\infty} \sigma(t, y) d t\right| d y
$$

For each $j \in \mathbb{N}$, set $I_{j}=\left[x, x+2^{j+1} h\right]$. Then,

$$
\begin{aligned}
& \leq \frac{1}{h} \int_{x}^{x+2 h}\left|\int_{x+4 h}^{\infty} \sigma(t, y) d t\right| d y \\
& \leq \frac{1}{h} \int_{x}^{x+2 h} \sum_{j=2}^{\infty} \int_{x+2^{j} h}^{x+2^{j+1} h}|\sigma(t, y)| d t d y \\
& \leq C \sum_{j=2}^{\infty} \int_{x+2^{j} h}^{x+2^{j+1} h}\left|b(t)-b_{J}\right|^{k}|f(t)| \frac{2 h}{\left(h\left(2^{j}-2\right)\right)^{2-\alpha}} d t \\
& \leq C \sum_{j=2}^{\infty} \frac{h^{\alpha}}{\left(2^{j-1}\right)^{1-\alpha}}\left(\frac{2}{2^{j-1} h} \int_{x+2^{j} h}^{x+2^{j+1} h}\left|b(t)-b_{J}\right|^{k}|f(t)| d t\right) \\
& \leq C \sum_{j=2}^{\infty} \frac{h^{\alpha}}{\left(2^{j-1}\right)^{1-\alpha}}\left(\frac{2}{2^{j-1} h} \int_{x+2^{j} h}^{x+2^{j+1} h}\left|b(t)-b_{I_{j}}\right|^{k}|f(t)| d t\right) \\
& \leq C \sum_{j=2}^{\infty} \frac{h^{\alpha}}{\left(2^{j-1}\right)^{1-\alpha}}(I V(x)+V(x)) .
\end{aligned}
$$

Let $\omega \in A^{+}\left(\frac{1}{\alpha}, \infty\right)$. Then $\omega^{\frac{-1}{1-\alpha}} \in A_{1}^{-}$. Choose $r>1$ such that $\omega^{\frac{-r}{1-\alpha}} \in$ $A_{1}^{-}$. Then, by Hölder's and John-Nirenberg's inequalities,

$$
\begin{aligned}
& I V(x) \leq\left(\frac{2}{2^{j-1} h} \int_{x+2^{j} h}^{x+2^{j+1} h}|f|^{\frac{1}{\alpha}} \omega^{\frac{1}{\alpha}}\right)^{\alpha} \\
& \quad \times\left(\frac{2}{2^{j-1} h} \int_{x+2^{j} h}^{x+2^{j+1} h}\left|b(t)-b_{I_{j}}\right|^{\frac{k}{1-\alpha}} \omega^{\frac{-1}{1-\alpha}}\right)^{1-\alpha} \\
& \leq C\left(2^{j} h\right)^{-\alpha}\|f \omega\|_{\frac{1}{\alpha}}\left(\frac{1}{2^{j} h} \int_{x}^{x+2^{j+1} h}\left|b(t)-b_{I_{j}}\right|^{\frac{k r^{\prime}}{1-\alpha}}\right)^{\frac{1-\alpha}{r^{\prime}}} \\
& \quad \times\left(\frac{1}{2^{j} h} \int_{x}^{x+2^{j+1} h} \omega^{\frac{-r}{1-\alpha}}\right)^{\frac{1-\alpha}{r}} \\
& \leq C\|b\|_{B M O}^{k}\left(2^{j} h\right)^{-\alpha}\|f \omega\|_{\frac{1}{\alpha}} \omega^{-1}(x) .
\end{aligned}
$$


Using again lemma 1 in [5] and Hölder's inequality,

$$
\begin{aligned}
V(x) \leq & \frac{1}{2^{j} h}\left|b_{I_{j}}-b_{J}\right|^{k} \int_{x}^{x+2^{j+1} h}|f(t)| d t \\
\leq & C(2 j)^{k}\|b\|_{B M O}^{k}\left(\frac{1}{2^{j} h} \int_{x}^{x+2^{j+1} h}|f|^{\frac{1}{\alpha}} \omega^{\frac{1}{\alpha}}\right)^{\alpha} \\
& \times\left(\frac{1}{2^{j} h} \int_{x}^{x+2^{j+1} h} \omega^{\frac{-1}{1-\alpha}}\right)^{1-\alpha} \\
\leq & C(2 j)^{k}\left(2^{j} h\right)^{-\alpha}\|b\|_{B M O}^{k}\|f \omega\|_{\frac{1}{\alpha}} \omega^{-1}(x) .
\end{aligned}
$$

Putting together inequalities (3.15), (3.16) and (3.17), we get that

$$
\begin{aligned}
& \frac{1}{h} \int_{x}^{x+2 h}\left|\int_{x+4 h}^{\infty} \sigma(t, y) d t\right| d y \\
& \leq C\|b\|_{B M O}^{k}\|f \omega\|_{\frac{1}{\alpha}} \omega^{-1}(x) \sum_{j=2}^{\infty}\left(\frac{2^{1-\alpha}}{2^{j}}+\frac{2^{1-\alpha}(2 j)^{k}}{2^{j}}\right) \\
& \leq C\|b\|_{B M O}^{k}\|f \omega\|_{\frac{1}{\alpha}} \omega^{-1}(x) .
\end{aligned}
$$

Taking supremums first on $h>0$ and then on $x \in \mathbb{R}$, we get

$$
\left\|\omega M_{5}^{+} f\right\|_{\infty} \leq C\|b\|_{B M O}^{k}\|f \omega\|_{\frac{1}{\alpha}} .
$$

So, by Theorem 3.2, for all $\omega \in A^{+}(p, q), \frac{1}{p}-\frac{1}{q}=\alpha$,

$$
\left\|M_{5}^{+} f\right\|_{\omega^{q}, q} \leq C\|b\|_{B M O}^{k}\|f\|_{\omega^{p}, p} .
$$

Proof of Theorem 2.10.

This proof also follows the same pattern as the preceding ones. Let $b \in B M O$ bounded and let $\lambda \in \mathbb{R}$. Then, as in (3.2), we have

$$
M_{\varphi, b}^{+, k} f(x) \leq M_{\varphi}^{+}\left((b-\lambda)^{k} f\right)(x)+\sum_{m=0}^{k-1} C_{k, m}|b(x)-\lambda|^{k-m} M_{\varphi, b}^{+, m} f(x) .
$$

Let us fix $x \in \mathbb{R}$ and $h>0$ and let $J=[x, x+8 h]$. Write $f=f_{1}+f_{2}$, where $f_{1}=f \chi_{J}$, and also write $\lambda=b_{J}$. Then, as in (3.3) and (3.12), it follows that

$$
\begin{aligned}
& \frac{1}{h} \int_{x}^{x+2 h}\left|M_{\varphi, b}^{+, k} f(y)-M_{\varphi}^{+}\left(\left(b-b_{J}\right)^{k} f_{2}\right)(x+2 h)\right| d y \\
& \leq \frac{1}{h} \int_{x}^{x+2 h}\left|M_{\varphi}^{+}\left(\left(b-b_{J}\right)^{k} f_{1}\right)(y)\right| d y \\
& \quad+\frac{1}{h} \int_{x}^{x+2 h}\left|M_{\varphi}^{+}\left(\left(b-b_{J}\right)^{k} f_{2}\right)(y)-M_{\varphi}^{+}\left(\left(b-b_{J}\right)^{k} f_{2}\right)(x+2 h)\right| d y \\
& \quad+\sum_{m=0}^{k-1} C_{k, m} \frac{1}{h} \int_{x}^{x+2 h}\left|b(y)-b_{J}\right|^{k-m}\left|M_{\varphi, b}^{+, m} f(y)\right| d y \\
& =I(x)+I I(x)+I I I(x) .
\end{aligned}
$$


It is clear that $I I I(x) \leq \sum_{m=0}^{k-1} C_{k, m} M_{3, m}^{+}\left(M_{\varphi, b}^{+, m} f\right)(x)$, being $M_{3, m}^{+}$the same as in the proofs of Theorems 2.8 and 2.9, and

$$
I I(x) \leq C \frac{1}{h} \int_{x}^{x+2 h} \int_{x+8 h}^{\infty} \frac{x+2 h-y}{(z-(x+2 h))^{2}}\left|b(z)-b_{J}\right|^{k}|f(z)| d z d y,
$$

by the conditions imposed on the kernel $\varphi$.

Consider the following sublinear operators defined on $\mathcal{C}_{c}^{\infty}$ :

$$
M_{6}^{+} f(x)=\sup _{h>0} \frac{1}{h} \int_{x}^{x+2 h}\left|M_{\varphi}^{+}\left(\left(b-b_{J}\right)^{k} f \chi_{J}\right)(y)\right| d y
$$

and

$$
M_{7}^{+} f(x)=\sup _{h>0} \frac{1}{h} \int_{x}^{x+2 h} \int_{x+8 h}^{\infty} \frac{x+2 h-y}{(z-(x+2 h))^{2}}\left|b(z)-b_{J}\right|^{k}|f(z)| d z d y,
$$

where, for each $h>0, J$ is the interval $[x, x+8 h]$.

The above inequalities and definitions give that

$$
\left(M_{\varphi, b}^{+, k} f\right)^{\#,+}(x) \leq C\left(M_{6}^{+} f(x)+M_{7}^{+} f(x)+\sum_{m=0}^{k-1} M_{3, m}^{+}\left(M_{\varphi, b}^{+, m} f\right)(x)\right) .
$$

Let $\omega \in A_{p}^{+}, 1<p<\infty$. Then, acting as in the boundedness of $M_{1}^{+}$, in the proof of Theorem 2.8, we get that $M_{6}^{+}$is bounded from $L^{p}(\omega)$ to $L^{p}(\omega)$. On the other hand, we already know that $M_{3, m}^{+}$is bounded from $L^{p}(\omega)$ to $L^{p}(\omega)$ and, to proceed with the induction, we observe that the case $k=0$ is a consequence of the fact that $M_{\varphi}^{+} f$ is pointwise equivalent to $M^{+} f$. Therefore, we only have to prove that $M_{7}^{+}$is bounded from $L^{p}(\omega)$ to $L^{p}(\omega)$, for $\omega \in A_{p}^{+}, 1<p<\infty$.

Let us use again Theorem 3.1. Let $\omega$ be such that $\omega^{-1} \in A_{1}^{-}$. For each $j \in \mathbb{N}$, let $I_{j}=\left[x, x+2^{j} h\right]$. Then

$$
\begin{aligned}
& \frac{1}{h} \int_{x}^{x+2 h} \int_{x+8 h}^{\infty} \frac{x+2 h-y}{(z-(x+2 h))^{2}}\left|b(z)-b_{J}\right|^{k}|f(z)| d z d y \\
& \leq C \frac{1}{h} \int_{x}^{x+2 h} h \sum_{j=3}^{\infty} \int_{x+2^{j} h}^{x+2^{j+1} h} \frac{\left|b(z)-b_{J}\right|^{k}}{(z-(x+2 h))^{2}}|f(z)| d z d y \\
& \leq C h|| f \omega \|_{\infty} \sum_{j=3}^{\infty} \frac{2^{j+1}}{\left(2^{j}-2\right)^{2} h^{2}} \frac{1}{2^{j+1}} \int_{I_{j}}\left|b(z)-b_{J}\right|^{k} \omega^{-1}(z) d z \\
& \leq C|| f \omega \|_{\infty} \sum_{j=3}^{\infty} \frac{2^{j+1}}{\left(2^{j}-2\right)^{2}}\left(\frac{1}{2^{j+1} h} \int_{I_{j}}\left|b(z)-b_{I_{j}}\right|^{k} \omega^{-1}(z) d z\right. \\
& =C\|f \omega\|_{\infty} \sum_{j=3}^{\infty} \frac{2^{j+1}}{\left(2^{j}-2\right)^{2}}(I V(x)+V(x)) .
\end{aligned}
$$


Let $q>1$ be such that $\omega^{-q} \in A_{1}^{-}$(Theorem 3.4). Then, Hölder's and John-Nirenberg's inequalities give

$$
\begin{aligned}
I V(x) & \leq\left(\frac{1}{2^{j+1} h} \int_{x}^{x+2^{j+1} h}\left|b-b_{I_{j}}\right|^{k q^{\prime}}\right)^{1 / q^{\prime}}\left(\frac{1}{2^{j+1} h} \int_{x}^{x+2^{j+1} h} \omega^{-q}\right)^{1 / q} \\
& \leq C\|b\|_{B M O}^{k} \omega^{-1}(x) .
\end{aligned}
$$

In [5], it is proved that $\left|b_{I_{j}}-b_{J}\right|^{k} \leq\left. C|2 j|^{k}|| b\right|_{B M O} ^{k}$ for all $3 \leq j$. Then, using this fact and using that $\omega^{-1} \in A_{1}^{-}$, we obtain

$$
V(x)=\frac{1}{2^{j+1} h} \int_{I_{j}}\left|b_{I_{j}}-b_{J}\right|^{k} \omega^{-1} \leq C|2 j|^{k}|| b \|_{B M O}^{k} \omega^{-1}(x) .
$$

Then by (3.20), (3.21) and (3.22) we get that, for all $\omega$ such that $\omega^{-1} \in$ $A_{1}^{-}$,

$$
\left\|\omega M_{7}^{+} f\right\|_{\infty} \leq C\|b\|_{B M O}^{k}\|f \omega\|_{\infty},
$$

and, by Theorem 3.1, for all $\omega \in A_{p}^{+}, 1<p<\infty$,

$$
\left\|M_{7}^{+} f\right\|_{\omega, p} \leq C\|b\|_{B M O}^{k}\|f\|_{\omega, p} .
$$

Now, we only have to take into account (3.19) and Theorem 3.3 to conclude the proof of Theorem 2.10 .

Remark 3.5. Following the same steps as in the proof of Theorem 2.10 we get Corollary 1 of [5], i.e., the boundedness of the commutator of onesided singular integrals (introduced in [1]), from $L^{p}(\omega)$ to $L^{p}(\omega), \omega \in A_{p}^{+}$, $1<p<\infty$.

Proof of Theorem 2.11 .

(iii) $\Rightarrow($ i) It is a consequence of Theorem 2.10.

$(i) \Rightarrow($ ii $)$ Let $p>1$ and set $\omega \equiv 1$.

$($ ii $) \Rightarrow($ iii $)$ Set $I=(a, b), I^{+}=(b, c)$, and $|I|=\left|I^{+}\right|$. Then

$$
\begin{aligned}
\frac{1}{|I|} \int_{I}\left|b(y)-b_{I^{+}}\right| d y & \leq\left(\frac{1}{|I|} \int_{I}\left|b(y)-b_{I^{+}}\right|^{k} d y\right)^{1 / k} \\
& =\left(\frac{1}{|I|} \int_{I}\left|\frac{1}{\left|I^{+}\right|} \int_{I^{+}}(b(y)-b(x)) d x\right|^{k} d y\right)^{1 / k} \\
& \leq\left(\frac{1}{|I|} \int_{I}\left(\frac{1}{\left|I^{+}\right|} \int_{I^{+}}|b(y)-b(x)|^{k} d x\right) d y\right)^{1 / k}
\end{aligned}
$$

Observe that, for $y \in I$,

$$
\begin{aligned}
\frac{1}{\left|I^{+}\right|} \int_{I^{+}}|b(x)-b(y)|^{k} d x & =\frac{1}{\left|I^{+}\right|} \int_{y}^{c}|b(x)-b(y)|^{k} \chi_{I^{+}}(x) d x \\
& \leq C M_{b}^{+, k} \chi_{I^{+}}(y) .
\end{aligned}
$$


Then, by Hölder's inequality and (ii),

$$
\begin{aligned}
\frac{1}{|I|} \int_{I} \mid b(y) & -b_{I^{+}} \mid d y \leq C\left(\frac{1}{|I|} \int_{I^{\prime}} M_{b}^{+, k} \chi_{I^{+}}(y) d y\right)^{1 / k} \\
& \leq C\left(\frac{1}{|I|} \int_{I}\left|M_{b}^{+, k} \chi_{I^{+}}(y)\right|^{p} d y\right)^{1 / p k} \\
& \leq C\left(\frac{1}{|I|} \int_{\mathbb{R}}\left|\chi_{I^{+}}(y)\right|^{p} d y\right)^{1 / p k} \leq C\left(\frac{\left|I^{+}\right|}{|I|}\right)^{1 / p k}=C .
\end{aligned}
$$

So $b \in B M O$.

Proof of Theorem 2.12. Observe that by the conditions given on $\varphi_{\alpha}, M_{\varphi_{\alpha}, b}^{+, k}$ can be treated in the same way as $I_{\alpha, b}^{+, k}$, the commutator of the one-sided fractional operator. Observe that the case $k=0$ is a consequence of the fact that $M_{\varphi_{\alpha}}^{+} f(x) \leq C M_{\alpha}^{+} f(x)$ and the result in [2].

Proof of Theorem 2.13.

$($ iii $) \Rightarrow(i)$ It is a consequence of Theorem 2.12 .

(i) $\Rightarrow($ ii $)$ Given an appropriate pair $(p, q)$, set $\omega \equiv 1$.

$($ ii $) \Rightarrow($ iii $)$ Set $I=(a, b), I^{+}=(b, c)$, and $|I|=\left|I^{+}\right|$. Then

$$
\begin{gathered}
\frac{1}{|I|} \int_{I}\left|b-b_{I^{+}}\right| d y \leq\left(\frac{1}{|I|} \int_{I}\left(\frac{1}{\left|I^{+}\right|} \int_{I^{+}}|b(x)-b(y)|^{k} d x\right) d y\right)^{1 / k} \\
=\left(\frac{|I|^{-\alpha}}{|I|} \int_{I}\left(\frac{1}{\left|I^{+}\right| 1^{-\alpha}} \int_{I^{+}}|b(x)-b(y)|^{k} d x\right) d y\right)^{1 / k} \cdot
\end{gathered}
$$

Observe that, for $y \in I$,

$$
\begin{aligned}
\frac{1}{\left|I^{+}\right| 1-\alpha} \int_{I^{+}}|b(x)-b(y)|^{k} d x & =\frac{1}{\left|I^{+}\right| 1-\alpha} \int_{y}^{c}|b(x)-b(y)|^{k} \chi_{I^{+}}(x) d x \\
& \leq C M_{\alpha, b}^{+, k} \chi_{I^{+}}(y) .
\end{aligned}
$$

Then, by Hölder's inequality and (ii),

$$
\begin{aligned}
\frac{1}{|I|} \int_{I}\left|b-b_{I^{+}}\right| d y & \leq C\left(\frac{|I|^{-\alpha}}{|I|} \int_{I} M_{\alpha, b}^{+, k} \chi_{I^{+}}(y) d y\right)^{\frac{1}{k}} \\
& \leq C\left(|I|^{-\alpha}\left(\frac{1}{|I|} \int_{I}\left|M_{\alpha, b}^{+, k} \chi_{I^{+}}(y)\right|^{q} d y\right)^{\frac{1}{q}}\right)^{\frac{1}{k}} \\
& \leq C\left(|I|^{-\alpha-\frac{1}{q}}\left(\int_{\mathbb{R}}\left|\chi_{I^{+}}(y)\right|^{p} d y\right)^{\frac{1}{p}}\right)^{\frac{1}{k}} \\
& \leq C\left(|I|^{-\alpha-\frac{1}{q}+\frac{1}{p}}\right)^{1 / k}=C .
\end{aligned}
$$

So $b \in B M O$.

Acknowledgement. We want to thank A. de la Torre and J.L. Torrea for their helpful comments and indications. 


\section{REFERENCES}

[1] H. Aimar, L. Forzani and F.J. Martín-Reyes, On weighted inequalities for one-sided singular integrals, Proc. Amer. Math. Soc. 125 (1997), 2057-2064.

[2] K. F. Andersen and E. T. Sawyer, Weighted norm inequalities for the RiemannLiouville and Weyl fractional integral operators, Trans. Amer. Math. Soc. 308 (1988), no. 2, 547-558.

[3] S. Bloom, A commutator theorem and weighted BMO, Trans. Amer. Math. Soc. 292 (1985), 103-122.

[4] R. Coifman, R. Rochberg, G.Weiss, Factorization theorems for Hardy spaces in several variables, Ann. of Math. 103 (2) (1976), 611-635.

[5] M. Lorente and M.S. Riveros, Weighted inequalities for commutators of one-sided singular integrals, Comment. Math. Univ. Carolinae 43 (1) (2002), 83-101.

[6] R. Macías and M.S. Riveros, One-sided extrapolation at infinity and singular integrals, Proc. Roy. Soc. Edinburgh Sect. A 130 (2000), 1081-1102.

[7] F.J. Martín-Reyes, New proofs of weighted inequalities for the one-sided HardyLittlewood maximal functions, Proc. Amer. Math. Soc. 117 (1993), 691-698.

[8] F.J. Martín-Reyes, P. Ortega and A. de la Torre, Weighted inequalities for onesided maximal functions, Trans. Amer. Math. Soc. 319 (2) (1990), 517-534.

[9] F.J. Martín-Reyes, L. Pick and A. de la Torre, $A_{\infty}^{+}$condition, Canad. J. Math. 45 (1993), 1231-1244.

[10] F.J. Martín-Reyes and A. de la Torre, Two weighted inequalities for fractional one-sided maximal operators, Proc. Amer. Math. Soc. 117 (2) (1993), 483-489.

[11] _ One-sided BMO spaces, J. London Math. Soc. 249 (1994), 529-542.

[12] B. Muckenhoupt, Weighted norm inequalities for classical operators, Proc. Sympos. Pure Math. Amer. Math. Soc., Providence, R.I. 35 (1979), no. 1, 69-83.

[13] C. Pérez, Endpoint estimates for commutators of singular integral operators, J. Funct. Anal. 128 (1) (1995), 163-185.

[14] Sharp estimates for commutators of singular integrals via iterations of the Hardy-Littlewood maximal function, J. Fourier Anal. Appl. 3 (6) (1997), 743-756.

[15] E. T. Sawyer, Weighted inequalities for the one-sided Hardy-Littlewood maximal functions, Trans. Amer. Math. Soc. 297 (1986), 53-61.

[16] C. Segovia and J.L. Torrea, Vector-valued commutators and applications, Indiana Univ. Math. J. 38 (4) (1989), 959-971.

[17] _ Weighted inequalities for commutators of fracctional and singular integrals., Publ. Mat. 35 (1991), 209-235.

[18] _ Higher order commutators for vector-valued Calderón-Zygmund operators., Trans. Amer. Math. Soc. 336 (2) (1993), 537-556.

[19] A. de la Torre and J.L. Torrea, One-sided discrete square function, Studia Math. 156 (3) (2003), 243-260.

Análisis Matemático. Facultad de Ciencias. Universidad de Málaga. (29071) Málaga, Spain

E-mail address: lorente@anamat.cie.uma.es

Famaf, Universidad Nacional de Córdoba, CiEM-Conicet. (5000) Córdoba, Argentina.

E-mail address: sriveros@mate.uncor.edu 